\title{
Research on the Transient Power Characteristics of the Inverter for Yun-Guang UHVDC
}

\author{
Chengjun Xia1, Haiwen Lan', Kun Men², Guokai Liang1 \\ ${ }^{1}$ School of Electric Power, South China University of Technology, Guangzhou, China \\ ${ }^{2}$ Electric Power Research Institute of CSG, China Southern Power Grid, Guangzhou, China \\ Email: 1037424860@qq.com
}

Received June 2014

\begin{abstract}
During the transient period of large-disturbance of the received power grid, the power features of the inverter are of great important for the security and stability of the system. The research before is defective in that the constant control mode assumption and the rough HVDC simulation model. The paper establishes the PSCAD/EMTDC model for Yun-Guang EHVDC system, and analysis the transient real power and the reactive power of the inverter. With the analysis of logic for control mode, the author also introduces the physical processes of the peak of the reactive power. At last, the paper puts forward several strategies for suppressing the peak of the dynamic reactive power and real power recovery acceleration.
\end{abstract}

\section{Keywords}

UHVDC, Inverter, Transient Power Features

\section{Introduction}

HVDC has huge transmission capacity and is a typical controlled system. HVDC is very sensitive to the fault of the inverter-side AC system. During the transient period of fault and recovery of the received power grid, the voltage disturbance of the inverter station may cause commutation failure in HVDC and lead to dynamic changes of reactive power and active power, while the power features of the inverter is of great important both for the rotor angle stability and voltage stability of the received system [1]-[4]. It's an economic strategy to optimize HVDC control system to accelerate the recovery of the dynamic real power, suppress the dynamic reactive power demand and even provide emergency power support [5] [6]. However, the existed studies on power characteristic of inverter are rare and have some shortcomings: firstly, the simulation models of inverter and HVDC control system adopted are not precise enough and most of the researches are done based on the electromechanical transient process; secondly, the analyses involving the exchange of inverter power are based on the constant control mode assumption. In fact, the large-disturbance will cause the drop of commutation voltage and may lead to a commutation failure in the inverter. The control mode switches to limit the DC current and 
may recover the commutation during the fault, maintaining a certain power transmission. As the fault is cleared, the AC voltage recovers, the irrational control system parameters may result in high dynamic reactive power demand and even lead to a subsequent commutation failure. During the transient period, the power features of the inverter, especially the dynamic reactive power feature is very complicated and must be studied combining with the analysis of its control dynamic behaviours.

This passage will firstly build the PSCAD/EMTDC model of Yun-Guang HVDC to analyse the power feature of the inverter during the transient period of fault and recovery and discuss its impact on the rotor angle stability and voltage stability of the received system. Lastly, this passage will set forth the physical process related to the peak of dynamic reactive power of the inverter combining with the switch logic of DC control mode and put forward several strategies for suppressing the peak of the dynamic reactive power and real power recovery acceleration.

\section{Simulation Study}

Firstly, we build the detailed model of Yun-Guang HVDC by means of PSCAD/EMTDC, whose main circuit parameters and control/protect models are consistent with the practical engineering. As we focus on the external characteristic of the converter, the AC system is represented as a Thevenin equivalent source (As it takes a long time for AC network regulating device to response, this power model is precise enough for the study on the transient process within 500 ms [7]). The simulation model is shown in Figure 1.

In order to make the simulation results more practical, the raw data is obtained from China Southern Power Grid under large power flow in some year, while Yun-Guang HVDC operates in bipolar mode with 5000 MW rated power and 2914MVar reactive power consumption of inverter in steady-state. The AC parameters can be achieved through multi-port Thevenin equivalent of sending and receiving end. The ESCRs of rectifier and inverter are calculated as 5.6 and 8.9 respectively, which indicates that the receiving system is strong enough to meet the reactive power demand of inverter during the transient dynamic process.

The simulation was taken in the DC system shown in Figure 1. A three-phase short circuit which lasted for $100 \mathrm{~ms}$ was set on the commutation bus of inverter. The different electrical distances between fault and commutation station were simulated by changing the ground impedance to obtain voltage drops in different degrees during the fault.

For the lack of space, only the Q-P transient feature will be explained here. In Figures 2(a)-(f), the horizontal axis represents the real power $P$ transmitted by inverter, while the vertical axis represents the reactive power $Q$ consumed by the inverter. P and Q are per-unit value (the rated power $500 \mathrm{MW}$ is the base value). The key turning points, their coordinates and also the moment when the working point arrives at them are marked in the figures above. Supposing that the system is working at point $\mathrm{O}$ when the fault occurs $(0 \mathrm{~ms})$, during the transient period, it moves from $\mathrm{O}$ to $\mathrm{A}$ to $\mathrm{B}$ to $\mathrm{C} \ldots$ and finally back to point $\mathrm{O}$ (real power has been recovered completely). Some conclusions are found through the figures above:

1) When the commutation failure doesn't occur, the fluctuation of the inverter power is small and the real power recovers fast $(270 \mathrm{~ms})$. Under critical condition(commutation failure doesn't occur exactly), the maximum real power loss is 0.1 p.u., while the peak reactive power demand which always appears during the fault is 0.08 p.u. more than the demand in steady-state.

2) When the commutation bus voltage drops to the range of 0.85 to 0.29 p.u. due to the disturbance of AC system, the power of inverter exhibits similar feature:

a) The feature of real power: (the fault occurs) the real power decreases quickly at first but decreases slowly later $\rightarrow$ the real power decreases to 0.4 p.u., the oscillation occurs and may cause reverse transfer power (depending on the severity of the voltage drop) $\rightarrow$ the real power stabilizes to a low value at $85 \mathrm{~ms}-90 \mathrm{~ms}$ (depending on the severity of the voltage drop) $\rightarrow$ (the fault is cleared) the real power increases quickly at first but increases slowly later, recovering overdampedly to the power value before the fault.

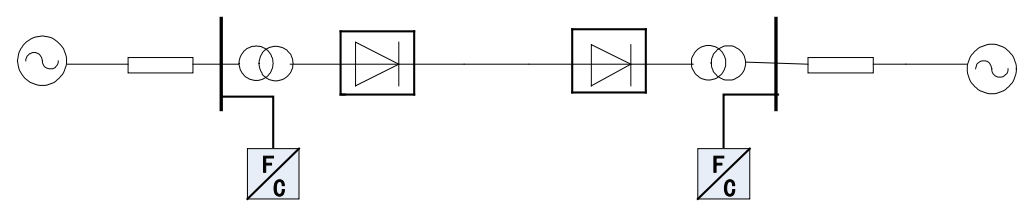

Figure 1. Diagram of the simulation model. 


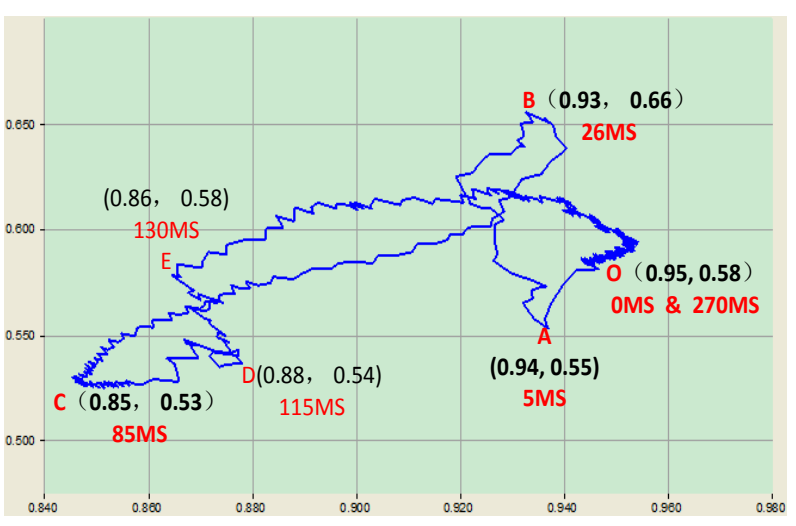

(a)

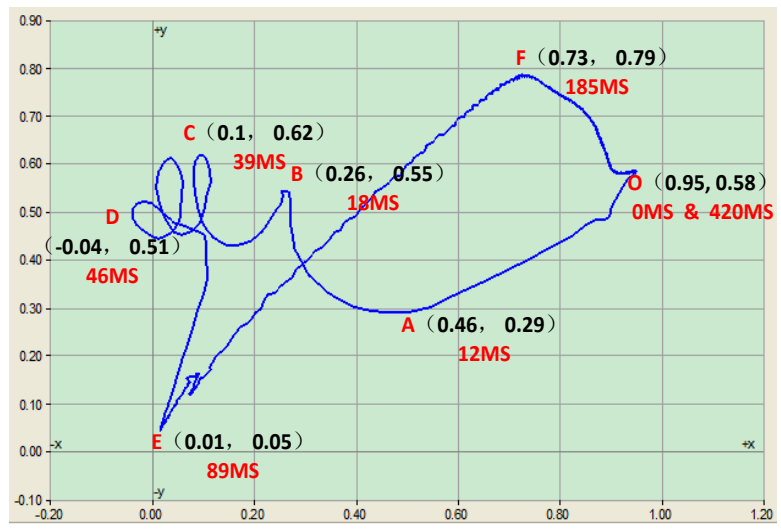

(c)

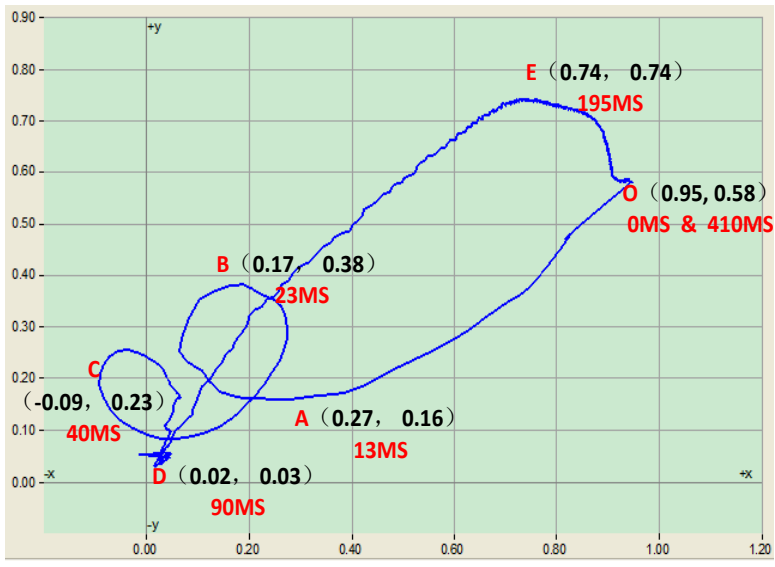

(e)

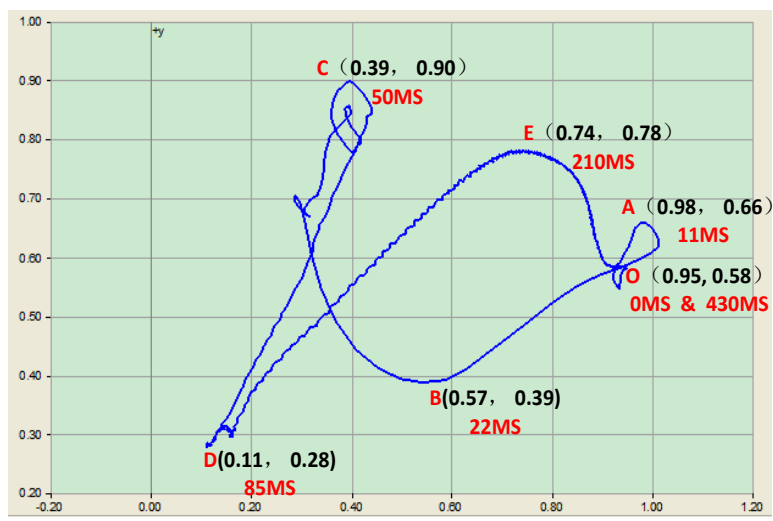

(b)

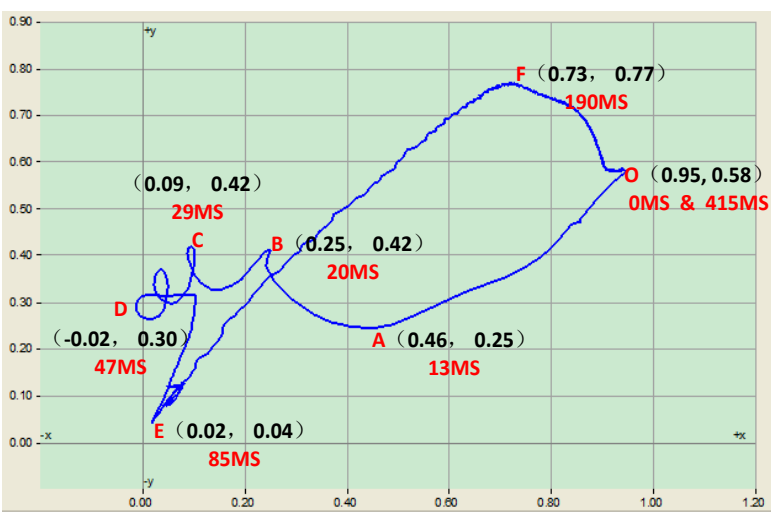

(d)

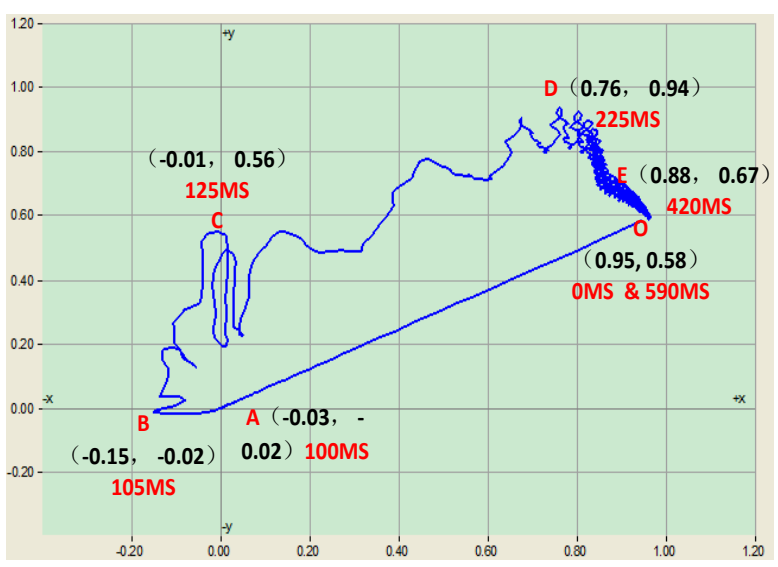

(f)

Figure 2. P-Q transient feature of inverter under different AC voltage drops. (a) Commutation bus voltage dropped to 0.91 p.u during the fault (commutation failure doesn't occur exactly); (b) Commutation bus voltage dropped to 0.85 p.u. during the fault (commutation failure occurs); (c) Commutation bus voltage dropped to 0.65 p.u. during the fault (commutation failure occurs); (d) Commutation bus voltage dropped to 0.48 p.u. during the fault (commutation failure occurs); (e) Commutation bus voltage dropped to 0.29 p.u. during the fault (commutation failure occurs); (f) A metallic short circuit occurred on the commutation bus (commutation failure).

b) The feature of reactive power: (the fault occurs) the reactive power decreases to the minimum value $\rightarrow$ the reactive power increases to the maximum value and may cause oscillation but the reactive power will never be transmitted reversely $\rightarrow$ the reactive power stabilizes to a low value $\rightarrow$ (the fault is cleared) when the real power recovers to about 0.74 p.u., the reactive power reaches to the peak value, and then decreases to the value before 
the fault.

c) The relationship between real power and reactive power: the real power and the reactive power have stabilized to a small range within 90 ms since the fault occurred and the power factor is relatively low. In the later recovery stage, reactive power becomes an important factor which restricts the recovery of real power. It can be confirmed by this phenomenon: as the reactive power nearly reaches to the peak value, the real power decreases quickly.

3) When a three-phase metallic short circuit occurs on the commutation bus, P and Q will drop to zero and never cause oscillation. When the fault is cleared, great real power may be transferred reversely and the reactive power increases with oscillation. As the commutation recover, the real power increases continuously, while the reactive power still oscillates. When the real power recovers to about 0.74 p.u., the reactive power reaches to the peak value of 0.94 p.u. and the restoration of DC power will be significantly decelerated.

In order to make the DC system have better operating performance, it's hoped that the real power of HVDC will recover as soon as possible in engineering to maintain the rotor angle stability between sending and receiving system and the reactive power will be consumed as little as possible to prevent the voltage instability of the receiving power grid. According to the analysis above, we can find that: $\mathrm{P}$ and $\mathrm{Q}$ are relevant to the dynamic feature of converter: the reactive power demand may reaches to two peak values. The peak value appears during the fault has great impact on the voltage stability of the AC grid, while the reactive power that capacitors of the converter station provide decreases because of the voltage drop, which means the convertors will consume great reactive power from the AC grid and drag the voltage down, leading to a vicious circle. During the recovery, the restoration of real power is restricted by the reactive power. The former $100 \mathrm{~ms}$ since the fault was cleared, the real power has increased quickly because of the abundant reactive power, while as the reactive power nearly reached to the peak (about 0.75 p.u.), the real power increased slowly. The following passage will focus on the physical process analysis related to the two peaks of dynamic reactive power, and put forward several strategies for suppressing the peak of the dynamic reactive power and real power recovery acceleration.

\section{Physical Process Analysis Related to the Peak of Dynamic Reactive Power}

The control system plays an important part in the operation of HVDC which influences the power and voltage [8]. The control mode will usually be switched many times during the fault and its recovery, so it's not appropriate to analyze based on a constant control mode assumption. The following passage will analyze the physical process related to dynamic reactive power peak combining with the switch logic of control system.

Though many new features are added to HVDC control system as the development of mordent control theory, the typical control feature is still be used in Yun-Guang HVDC control system because of the conservatism of power industry. The control mode of the rectifier includes constant current control (including VDCOL) and constant $\alpha_{\min }$ control while the control mode of the inverter includes constant voltage control, constant $\gamma$ control, constant current control (including VDCOL) and constant $\alpha_{\min }$ control. The control instructions of control layers above pole control such as power modulation are not considered for the following two reasons: 1) the time constant of pole control instruction is high; 2) the minimum current value is chosen from all current instructions in pole control. The current value set by the power control instruction won't be chosen since it is high. Though the control mode is chosen automatically in valve control layer (rectifier chooses the minimum value, while inverter chooses the maximum value), the control mode switch logic during the transient period of large-disturbance is shown in Figure 3 according to a lot of simulation results. The inverter power expression is derived below:

$$
\left\{\begin{array}{l}
P_{d i}=U_{d i} I_{d}=\left(K_{m} E_{i} \cos \gamma_{\mathrm{i}}-R_{c i} I_{d}\right) I_{d} \\
Q_{d i}=I_{d} \sqrt{\left(K_{m} E_{i}\right)^{2}-U_{d i}^{2}}=I_{d} \sqrt{\left(K_{m} E_{i}\right)^{2}-\left(K_{m} E_{i} \cos \gamma_{i}-R_{c i} I_{d}\right)^{2}}
\end{array}\right.
$$

$P_{d i}$ and $Q_{d i}$ are respectively the real power the converters transmit and reactive power the converters consume. $E_{i}, U_{d i}, I_{d}, \gamma_{i}$ are respectively the AC voltage, DC voltage, DC current and extinction angle of inverter. $K_{m}$ and $R_{c i}$ are constant values.

\subsection{The First Peak of Dynamic Reactive Power}

The fault may cause an inverter commutation failure which leads to the decrease of $U_{d c}$ in inverter and the increase of $I_{d}$. It can be found in Figure 3 that inverter will choose the constant $\gamma$ control mode which will increase $\beta$ rapidly. Since the voltage of commutation bus doesn't drop to zero during the fault, the inverter will recover 


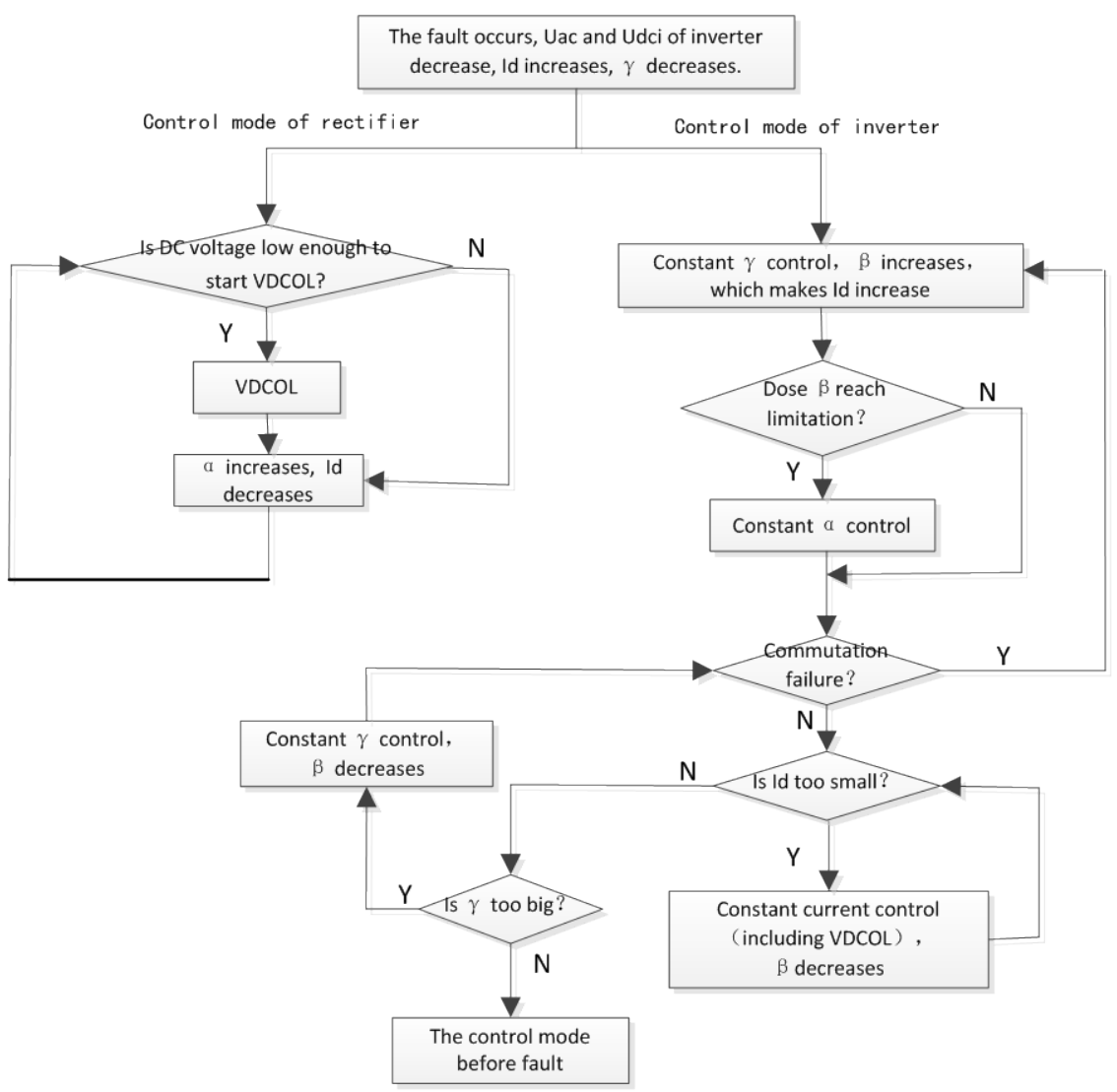

Figure 3. The control mode switch logic during the transient period of large-disturbance.

commutation rapidly. As $\gamma$ usually overshoots to a high value because of the inherent characteristic of PI controller and the current value is high this time, the first peak value appears according to expression (1).

Here is the corresponding control strategy. It's easy to found that:

$$
\frac{\partial Q_{d i}}{\partial E_{\bar{i}}}>0 ; \frac{\partial Q_{d_{\bar{d}}}}{\partial \gamma_{\mathrm{i}}}<0 ; \frac{\partial Q_{d_{i}}}{\partial I_{d}}>\mathbf{0}
$$

During the fault, AC voltage $E_{i}$ is small, which is benefit to the suppression of the peak value of the dynamic reactive power, but it depends on the severity of the fault. Though the decrease of $\gamma$ will lead to the decrease of reactive power, the high $\gamma$ value during the commutation recovery caused by the overshoot of control system is not suitable to be altered, or may increase the duration of commutation failure. It's suitable to decrease the DC current $I_{d}$ by alter the parameters of VDCOL, whose parameters play an important in dynamic power [9].

\subsection{The Second Peak of Dynamic Reactive Power}

The second peak value appears in the period of recovery of DC system and it's supposed that commutation failure won't occur. When the fault is cleared, the DC voltage increases as the AC voltage increases. According to Figure 3, we can find that the inverter chooses constant $\gamma$ control at this time to decrease the high $\gamma$ caused by overshoot. So the change of voltage $E_{i}$, DC current $I_{d}$ and $\gamma$ during the recovery are benefit to the increase of reactive power. Since the voltage recovers more quickly than the current because of the delay link of VDCOL, the peak value of the reactive power will appears at the moment when the current reaches to the peak. In addition, according to the analysis of control system, we can find that Yun-Guang HVDC control system has adopted such a strategy to avoid commutation failure: regulating $\beta$ rapidly if $\gamma$ is small, regulating $\beta$ slowly if $\gamma$ is big. This is why the reactive power increases slowly during the later period of recovery.

Here is the corresponding control strategy. In order to suppress the peak of the reactive power, we can take measures to suppress the overshoot of current and increase the drop rate of $\gamma$. The latter is also benefit to the re- 
covery of real power, but may increase the risk of commutation failure, so it must be carefully considered. The former can be achieved through optimizing the parameters of VDCOL. When the VDCOL control starts, we can find the following equation:

$$
K_{m} E_{i} \cos \gamma_{i}-R_{c i} I_{d}=K I_{d}+B
$$

The left side of the equation depends on the converter, while the right side depends on the VDCOL curve. $K$ is the slope, $B$ is the intercept. The value of $I_{d}$ can be solved as below:

$$
I_{d}=\frac{1}{R_{c i}+K}\left(K_{m} E_{i} \cos \gamma_{i}-B\right)
$$

We can find that the current is not only relevant to AC voltage $E_{i}$ but also VDCOL parameters $K$ and $B$. The transient power feature optimization can usually be achieved by altering these two parameters.

\section{Conclusions}

When the disturbance of the AC system is not large enough to cause commutation failure, the fluctuation of the real power is small and the real power recovers fast.

When the disturbance of the AC system is large enough to cause commutation failure, the P-Q transient curves of inverter under different AC voltage drops are similar. P and Q stabilize to a low value at $85 \mathrm{~ms}-90 \mathrm{~ms}$ after the fault occurs. The reactive power reaches to the peak value (about 0.74 p.u.) at about $100 \mathrm{~ms}$ after the fault is cleared. Later, the real power increases significantly slowly.

The study on power features of the inverter is benefit both to the rotor angle stability and voltage stability of the received system. In most cases, the peak demand of dynamic reactive power of inverter usually appears respectively during the period of fault and recovery. According to the theoretical analysis, the peak suppression of the dynamic reactive power and the real power recovery acceleration can be achieved by optimizing the VDCOL link, PI controller and constant $\gamma$ control.

\section{Acknowledgements}

This work was financially supported by National High Technology Research and Development Program of China (2012AA050209).

\section{References}

[1] Zhang, J.S., Zhang, Y., Zhang, Z.C. and Wu, Z.G. (2005) Influence of DC System Control Modes on Voltage and Power Recovery after Large Disturbance in Hybrid AC/DC System. Power System Technology, 20, 20-24.

[2] Yang, W.D., Xue, Y.S., Jing, Y., Chao, J., Huang, W.L., Hang, C. and Yang, B.B. (2003) A Coordinated Recovery Strategy for 3 HVDC System Involved in the South China Power Grid. Automation of Electric Power Systems, 27, 6770.

[3] Chen, X.Y., Han, M.X. and Liu, C.R. (2012) A Coordinate Rotational Transformation Based Virtual Power V/f Droop Control Method for Low Voltage Microgrid. Automation of Electric Power Systems, 36, 58-63.

[4] Zhang, Y.D. (2013) Research on Dynamic Reactive Power Demand Characteristics and Counter-Measures of Received Power Grid in AC/DC Hybrid Power System.

[5] Chen W.-B., Zhang, Y. and Xie, H.-F. (2010) Study on Emergency DC Power Support for UHVDC Fault. Proceedings of the CSU-EPSA, 22, 113-118.

[6] Guo, Q.W. (2012) Study on the Dynamic Characteristic of $\pm 660 \mathrm{kV}$ Yidong HVDC System.

[7] CIGRE WG 14.07, IEEE WG 15.05 (1997) Guide for Planning DC Links Terminating at AC Locations Having Low Short-Circuit Capacities. 120-121.

[8] Liu, X.M. (2011) Influence of DC System Control Mode on Voltage Stability of Receiving-End Power Grid. Electric Power Automation Equipment, 31, 69-77.

[9] Wang, K. (2007) Study of VDCOL Control Strategies for Coordinated Recovery of Multi-feed-in HVDC System. Jiangsu Electrical Engineering, 26, 1-4. 